\title{
Solid lipid nanoparticles for thermoresponsive targeting: evidence from spectrophotometry, electrochemical, and cytotoxicity studies
}

This article was published in the following Dove Press journal:

International Journal of Nanomedicine

Number of times this article has been viewed

\author{
Mubashar Rehman ${ }^{1-3}$ \\ Ayesha Ihsan ${ }^{2}$ \\ Asadullah Madni' \\ Sadia Zafar Bajwa ${ }^{2}$ \\ $\mathrm{DiShi}$ \\ Thomas J Webster ${ }^{3,4}$ \\ Waheed S Khan ${ }^{2}$
}

'Department of Pharmacy, The Islamia University of Bahawalpur, Bahawalpur, Punjab, Pakistan; ${ }^{2}$ Nanobiotech Group, National Institute of Biotechnology and Genetic Engineering, Faisalabad, Punjab, Pakistan; ${ }^{3}$ Department of Chemical Engineering, Northeastern University, Boston, MA, USA; ${ }^{4}$ Center of Excellence for Advanced Materials Research, King Abdulaziz University, Jeddah, Saudi Arabia
Correspondence: Ayesha Ihsan Nanobiotech Group, National Institute of Biotechnology and Genetic Engineering, Jhang Road, Faisalabad 38000, Punjab, Pakistan

$\mathrm{Tel}+92419201316$

Fax +92 4| 920 |472

Email aishaehsan@gmail.com

Asadullah Madni

Department of Pharmacy, The Islamia University of Bahawalpur, Railway Road, Bahawalpur 63100, Punjab, Pakistan

Tel +92 629255243

Fax +92629255565

Email asadpharmacist@hotmail.com
Abstract: Thermoresponsive drug delivery systems are designed for the controlled and targeted release of therapeutic payload. These systems exploit hyperthermic temperatures $\left(>39^{\circ} \mathrm{C}\right)$, which may be applied by some external means or due to an encountered symptom in inflammatory diseases such as cancer and arthritis. The objective of this paper was to provide some solid evidence in support of the hypothesis that solid lipid nanoparticles (SLNs) can be used for thermoresponsive targeting by undergoing solid-liquid phase transition at their melting point (MP). Thermoresponsive lipid mixtures were prepared by mixing solid and liquid natural fatty acids, and their MP was measured by differential scanning calorimetry (DSC). SLNs (MP $39^{\circ} \mathrm{C}$ ) containing 5 -fluorouracil (5-FU) were synthesized by hot melt encapsulation method, and were found to have spherical shape (transmission electron microscopy studies), desirable size $(<200 \mathrm{~nm})$, and enhanced physicochemical stability (Fourier transform infrared spectroscopy analysis). We observed a sustained release pattern $(22 \%-34 \%)$ at $37^{\circ} \mathrm{C}$ ( 5 hours). On the other hand, $>90 \%$ drug was released at $39^{\circ} \mathrm{C}$ after 5 hours, suggesting that the SLNs show thermoresponsive drug release, thus confirming our hypothesis. Drug release from SLNs at $39^{\circ} \mathrm{C}$ was similar to oleic acid and linoleic acid nanoemulsions used in this study, which further confirmed that thermoresponsive drug release is due to solid-liquid phase transition. Next, a differential pulse voltammetry-based electrochemical chemical detection method was developed for quick and real-time analysis of 5-FU release, which also confirmed thermoresponsive drug release behavior of SLNs. Blank SLNs were found to be biocompatible with human gingival fibroblast cells, although 5-FU-loaded SLNs showed some cytotoxicity after 24 hours. 5-FU-loaded SLNs showed thermoresponsive cytotoxicity to breast cancer cells (MDA-MB-231) as cytotoxicity was higher at $39^{\circ} \mathrm{C}$ (cell viability $72 \%-78 \%$ ) compared to $37^{\circ} \mathrm{C}$ (cell viability $>90 \%$ ) within 1 hour. In conclusion, this study presents SLNs as a safe, simple, and effective platform for thermoresponsive targeting.

Keywords: temperature sensitive, breast cancer, 5-fluorouracil, nanostructured lipid carriers, emulsions, fatty acids

\section{Introduction}

Thermoresponsive triggered release of the potent chemotherapeutic drugs has shown promising results in various research reports and clinical trials. ${ }^{1}$ Thermoresponsive drug delivery systems (TDDS) are sensitive to higher temperature $\left(39^{\circ} \mathrm{C}-45^{\circ} \mathrm{C}\right)$ and release payload at target sites, ie, hyperthermic body tissues. ${ }^{2}$ However, synthesis of the thermoresponsive hydrogels and liposomes generally involves synthesis of block copolymers via complex chemical reactions and use of potentially toxic reagents. ${ }^{3,4}$ Liposomes are most widely used for thermoresponsive targeting, and one 
thermoresponsive liposome formulation, ie, ThermoDox ${ }^{\circledR}$, has gained the US Food and Drug Administration (FDA) approval for the treatment of lung cancer. ${ }^{5}$ ThermoDox ${ }^{\circledR}$ is administered in combination with the radiofrequency ablation for $>45$ minutes. Radiofrequency ablation is applied to kill major portion of the tumor and also induces release of doxorubicin to kill remaining tumor mass. However, ThermoDox ${ }^{\circledR}$ has short circulation life and should be administered immediately before radiofrequency ablation. ${ }^{1}$

Many embodiments of thermoresponsive liposomes show low encapsulation efficiency $(\mathrm{EE})^{6}$ and unpredictable drug release, ${ }^{3,7}$ and their blood circulation life is very short. ${ }^{1,8}$ On the other hand, solid lipid nanoparticles (SLNs) have been reported to show long circulation life ( $>24$ hours) and passively target cancers by enhanced permeability and retention (EPR) effect. ${ }^{9,10}$ In addition to this, SLN composition and their methods of preparation are considered safe as compared to other novel DDS such as liposomes. ${ }^{11}$

Previously, SLNs have been used in combination with hydrogels to achieve thermoresponsive drug delivery. ${ }^{12}$ In this system, hydrogels act as a thermoresponsive component and SLNs are used as a drug reservoir. Recently, we have reported for the first time that SLNs can be used for thermoresponsive drug delivery by tuning their melting point (MP). ${ }^{13}$ These SLNs are solid at room temperature $\left(37^{\circ} \mathrm{C}\right)$ and show minimal drug release. However, at the hyperthermic temperature $\left(>39^{\circ} \mathrm{C}\right)$, they melt and undergo solid-liquid phase transition, leading to faster diffusion of drug into the surrounding dissolution medium. ${ }^{13,14}$ Since then, other research groups have also explored thermoresponsive drug release from SLNs. For example, ud Din et al prepared thermoresponsive SLN-loaded hydrogels for rectal delivery of flurbiprofen. These SLNs remained solid during storage conditions and underwent solid-liquid phase transition (MP $32^{\circ} \mathrm{C}$ ) at physiological temperature. ${ }^{15}$ In comparison to our reported thermoresponsive SLNs, these nanoparticles would be liquid at normal body temperature and cannot be regarded as TDDS. More recently, Brezaniova et al have reported temoporfin-loaded thermoresponsive SLN for photodynamic therapy of cancer using 1-tetradecanol lipids that melt at $39^{\circ} \mathrm{C}$. Although they showed superior photodynamic efficacy against cancer, the critical parameters such as drug release, cytotoxicity or in vivo anticancer activity as a function of temperature $\left(37^{\circ} \mathrm{C}\right.$ and $\left.39^{\circ} \mathrm{C}\right)$ are still not available. ${ }^{6}$

While designing a smart DDS, periodic drug release is mostly studied by using the dissolution apparatus, which involves removal of samples from the dissolution medium. This protocol requires adjustment of equations to accommodate change in volume or the amount of the drug removed along with samples. Moreover, the samples should be analyzed either by the ultraviolet (UV)-Vis/fluorescence spectrophotometry or by high-performance liquid chromatography (HPLC), which is a laborious and time-consuming procedure. Therefore, an easy, quick and robust method is needed to quantify drug released from drug delivery systems. The differential pulse voltammetry (DPV) method has been used previously for assay of 5-fluorouracil (5-FU) in pharmaceutical dosage forms. ${ }^{16}$ It involves application of the regular voltage pulses of increasing amplitude, which causes oxidation of the analyte and generation of current. The potential at which oxidation peak appears is characteristic of the analyte, and the height of the peak is directly related to the concentration of the analyte. Thus, DPV can be used for the qualitative and quantitative determination of electrochemically active drugs such as 5-FU.

The need of safe and effective targeted drug delivery systems can be addressed by SLNs. Therefore, this study was designed to comprehensively elucidate SLNs as alternatives to conventional TDDS. Different thermoresponsive lipid mixtures (TLMs) were prepared that would melt at $39^{\circ} \mathrm{C}$ and fabricate into SLNs. Characterization of solid-liquid phase transition in SLNs and drug release from SLNs were studied as a function of temperature. Furthermore, a DPV method was also developed for real-time determination of 5-FU release from the SLNs at physiological $\left(37^{\circ} \mathrm{C}\right)$ and hyperthermic conditions $\left(39^{\circ} \mathrm{C}\right)$. Then, in vitro cytotoxicity studies were performed at $37^{\circ} \mathrm{C}$ and $39^{\circ} \mathrm{C}$ to confirm thermoresponsive nature of SLNs. To the best of our knowledge, this is the first report to comprehensively characterize thermoresponsive behavior of SLNs and provide a novel method for the realtime monitoring of stimuli-responsive drug release.

\section{Methodology Materials}

Lauric acid (LA), oleic acid (OA), linoleic acid (LIA), Brij 58, Span 80, ethanol and 5-FU were purchased from Sigma-Aldrich Co. (St Louis, MO, USA). Dialysis bags (molecular weight cutoff value 8,000 Da) were purchased from Spectrum Labs (Rancho Dominguez, Canada). Tetrazolium dye (MTS) reagent for cell viability assay was purchased from Promega Corporation (Fitchburg, WI, USA). Human gingival fibroblast (HGF; PCS-201-108) cells and human breast adenocarcinoma cells (MDA-MB-231, HTB-26) were purchased from American Type Culture Collection (ATCC) (Manassas, VA, USA). Dulbecco's Modified Eagle's Medium (DMEM) was purchased from GE Healthcare UK Ltd (Little Chalfont, UK). Milli Q deionized water $(\sim 18.2 \mu \mathrm{S})$ was used throughout the study. 


\section{Preparation and characterization of lipid mixtures}

LA (12 carbon atoms, MP $44^{\circ} \mathrm{C}$ ) was selected as the solid fatty acid and mixed with either of liquid fatty acids, ie, OA (MP $14^{\circ} \mathrm{C}$ ) or LIA $\left(\mathrm{MP}-5^{\circ} \mathrm{C}\right)$, in different ratios $(3: 1-12: 1)$. First, LA was melted to $50^{\circ} \mathrm{C}$, and liquid fatty acid was added to it. Lipids were mixed for 5 minutes and cooled down to room temperature to form lipid mixture of LA and OA (LO) and LA and LIA (LL). These lipid mixtures were characterized by differential scanning calorimetry (DSC) to determine MP. Then, a graph was plotted between MP of the lipid mixtures and the proportion of solid fatty acid in the lipid mixture. Straight line equations were derived for the graphs to determine the proportion of solid fatty acids for TLM with MP of $39^{\circ} \mathrm{C}$.

\section{Evaluation of solid-liquid phase transition by light transmission studies}

It is widely accepted that solid lipids are opaque, whereas oils are transparent to light. Therefore, light transmission studies were performed at $37^{\circ} \mathrm{C}$ and $39^{\circ} \mathrm{C} .{ }^{17}$ Briefly, lipid mixture was taken in a cuvette of UV-Vis spectrophotometer (U2020; IRMECO, Geesthacht, Germany) and reading was measured at $500 \mathrm{~nm}$ at $37^{\circ} \mathrm{C}$ and $39^{\circ} \mathrm{C}$.

\section{Preparation of SLNs}

SLNs can be prepared by various methods. ${ }^{18}$ Previously, we have reported that the hot melt encapsulation (HME) method is superior to other methods due to the lack of toxic organic solvents and the ease of process parameters. ${ }^{14}$ Therefore, HME was selected for the preparation of the SLNs with composition shown in Table 1. First, TLM (100 mg) was melted to $45^{\circ} \mathrm{C}$ and 5 -FU was added to it. Stirring was continued for 5 minutes, and aliquot of ethanol was added to facilitate homogenous mixing of 5-FU in the lipid phase. Aqueous phase $(5 \mathrm{~mL})$ was prepared by mixing either Brij 58 surfactant $(5 \%)$ alone or the combination of Brij 58 and
Span 80 (3.75\% and $1.25 \%$, respectively) in water and heated to $45^{\circ} \mathrm{C}$. Then, the lipid phase was added drop wise to the aqueous phase and stirring was continued at $45^{\circ} \mathrm{C}$. After 5 minutes, the heater of the hot plate stirrer was switched off and the hot melt emulsion so formed was allowed to cool down to room temperature. The as-prepared SLNs were centrifuged at $14,000 \mathrm{rpm}$ at $4^{\circ} \mathrm{C}$ and washed with deionized water three times following the same procedure. SLNs were lyophilized in the presence of ethylene glycol as a cryoprotectant at $-105^{\circ} \mathrm{C}$ and reduced pressure for 24 hours.

\section{Characterization of SLNs}

Thermoresponsive nature of the SLNs was confirmed by the light transmission studies at different temperatures $\left(37^{\circ} \mathrm{C}\right.$, $38^{\circ} \mathrm{C}$ and $39^{\circ} \mathrm{C}$ ) as described earlier. The size and zeta potential of SLNs were measured by using zetasizer (ZS 90; Malvern Instruments, Malvern, UK). The morphology of SLNs was studied by transmission electron microscopy (TEM; JSM 75000; JEOL, Tokyo, Japan).

Yield of the SLNs produced by a method is required from pharmaceutical point of view. In this study, the SLNs produced in a single batch were weighed, and the percentage yield was calculated by using the following formula: ${ }^{19}$

Percentage yield

$$
=\frac{\text { Actual weight of nanoparticles produced }}{\text { Theoretical weight of nanoparticles produced }}
$$

Stability of 5-FU and its compatibility with excipients was evaluated by attenuated total reflection-Fourier-transform infrared (FTIR) spectroscopy. FTIR spectra were taken for all individual components, 5-FU and drug-loaded SLNs. In case of any chemical interaction, 5-FU will not be released in dissolution studies or its efficacy may be lost in the cytotoxicity studies. Existence of intact 5-FU peaks in the SLNs would indicate its compatibility with other excipients. ${ }^{20}$ The

Table I Composition and properties of SLNs

\begin{tabular}{|c|c|c|c|c|c|c|c|}
\hline $\begin{array}{l}\text { Formulation } \\
\text { code }\end{array}$ & $\begin{array}{l}\text { Lipid } \\
\text { mixture }\end{array}$ & Surfactant & 5-FU (mg) & Size $(n m)$ & PDI & $\begin{array}{l}\text { Zeta } \\
\text { potential }\end{array}$ & EE (\%) \\
\hline FLOM & LO & Brij 58 & 5 & 120 & 0.2 & -34.0 & 42.32 \\
\hline FLOD & LO & Brij 58:Span 80 & 5 & 198 & 0.2 & -36.2 & 40.36 \\
\hline FLLM $^{a}$ & LL & Brij 58 & 5 & - & - & - & - \\
\hline FLLD & LL & Brij 58:Span 80 & 5 & 158 & 0.1 & -40.5 & 40.12 \\
\hline
\end{tabular}

Notes: aFLLM was unstable and immediately formed a gel-like phase. Therefore, its characterization was not performed.

Abbreviations: SLN, solid lipid nanoparticle; 5-FU, 5-fluorouracil; PDI, polydispersity index; EE, encapsulation efficiency; FLOM, 5-FU-loaded lauric acid and oleic acid nanoparticles prepared with mono-surfactant system; LO, lauric acid and oleic acid; FLOD, 5-FU-loaded lauric acid and oleic acid nanoparticles prepared with doublesurfactant system; FLLM, 5-FU-loaded lauric acid and linoleic acid nanoparticles prepared with mono-surfactant system; LL, lauric acid and linoleic acid; FLLD, 5-FU-loaded lauric acid and linoleic acid nanoparticles prepared with double-surfactant system. 
colloidal stability of SLNs was measured in phosphate-buffered saline (PBS) (1, 0.1 and $0.01 \mathrm{M})$ and plasma. SLNs were dispersed in each solution and stored at ambient temperature. After 24 hours, SLN dispersions were sonicated for 1 minute and size was evaluated by DLS measurements. ${ }^{21}$

\section{Drug loading studies}

Calibration curve was constructed using standard solutions to find unknown concentration of samples. ${ }^{22} \mathrm{EE}$ is the amount of drug encapsulated in nanoparticles from a given amount of drug used. In other words, it is percent efficiency of nanoparticles to contain drug. First, nanoparticles were separated by ultracentrifugation. After completion, the supernatant was taken and its absorbance was recorded at $258 \mathrm{~nm}$ to measure the amount of drug in it using the calibration curve. Then, EE was calculated by the following formula:

$$
\begin{aligned}
& \text { Encapsulation efficiency }(\%) \\
& \qquad=\frac{\text { Weight of drug encapsulated }}{\text { Total weight of drug added }} \times 100
\end{aligned}
$$

\section{Drug release studies by UV-Vis spectrophotometer}

The dissolution studies are used to surrogate in vivo drug release behavior. In this study, the dialysis bag method was employed by using United States Pharmacopeia (USP) type II dissolution apparatus with paddle assembly (Pharma Test, Hainburg, Germany). ${ }^{14,23}$ Cellulose ester dialysis bag (molecular weight cutoff value $10,000 \mathrm{Da}$ ) was selected on the basis of molecular weight of drug and size of nanoparticles. The SLNs equivalent to $4 \mathrm{mg}$ 5-FU were taken in a dialysis bag and tied at both ends. The filled dialysis bags were placed in the vessel of the dissolution apparatus containing $250 \mathrm{~mL}$ dissolution medium, ie, PBS, of $\mathrm{pH} 7.4 .{ }^{24}$ Dissolution studies were carried out by adjusting the paddle speed at $50 \mathrm{rpm}$, and the temperature was set to $37^{\circ} \mathrm{C}$ and $39^{\circ} \mathrm{C}$ for the evaluation of the thermoresponsive drug release. Samples were taken out at predetermined time intervals, and their absorbance was taken by UV-Vis spectrophotometer (U2020). Absorbance was fitted to the straight line equation of the calibration curve to find the amount of the 5-FU released.

\section{Dissolution studies by DPV}

The DPV setup for drug release studies consisted of a silver/ silver chloride reference electrode, a platinum counter electrode and a graphite working electrode (Figure 1). First, calibration curve was constructed by adding different amounts of 5 -FU (0.05-5 mM) in $25 \mathrm{~mL}$ of PBS (0.1 M, pH 7.4) taken in a glass flask. The electrodes were dipped in the PBS-containing flask, and readings were taken using Autolab (PGStat 30; Metrohm, Riverview, FL, USA). Next, the SLNs containing 5-FU (equivalent to $1 \mathrm{mM}$ ) were taken in the dialysis bag and placed in the $25 \mathrm{~mL}$ of the PBS (pH 7.4). Readings were taken at different time intervals as the electrodes were kept in the dissolution medium throughout the experiment. The amount of 5-FU released was calculated using the calibration curve. In all runs, samples were purged with nitrogen gas to degas and remove oxygen.

\section{Dissolution kinetics studies}

Dissolution profiles obtained by the UV-Vis spectrophotometric method and electrochemical method were analyzed by model-independent and model-dependent approaches as recommended by the FDA for comparison of drug release. Model independent analysis included calculation of difference factor $\left(f_{1}\right)$ and similarity factors $\left(f_{2}\right) . f_{1}$ calculates percent error, while $f_{2}$ represents percentage similarity between two curves. Generally, $f_{1}$ value is $<15$ and $f_{2}$ value is $>50$ to ensure equivalence in two drug release data sets. ${ }^{25}$

Model dependent analysis was done by fitting dissolution profiles in first-order, zero-order, Korsmeyer-Peppas and Higuchi models. ${ }^{26}$ The analysis was done by using DDSolver, an extension of MS Excel.

\section{Cytotoxicity studies}

HGF cell lines were used as normal cells, whereas MDAMB-231 cell line was used as a model breast cancer cell line. Cells were defrosted by thawing in warm water and cultured in the DMEM containing 10\% fetal bovine serum and $1 \%$ penicillin/streptomycin in a $75 \mathrm{~cm}^{2}$ flask. The incubator was set at $37^{\circ} \mathrm{C}$ and $5 \%$ carbon dioxide. Cells were subcultured two times and used for further studies when they reached $80 \%$ confluence.

Biocompatibility and cytotoxicity studies were performed against normal tissue cell line (HGF) and breast cancer cell line (MDA-MB-231). Cells were seeded at a density of 15,000 cells $/ \mathrm{cm}^{2}$ in a 96 -well plate using the DMEM containing $10 \%$ fetal bovine serum and $1 \%$ penicillin/streptomycin. After 24 hours incubation, samples were added to each cell line with drug concentration ranging from 0.05 to $5 \mu \mathrm{M}$ for HGF and 0.05 to $1 \mu \mathrm{M}$ for MDA-MB-231, respectively. For HGF biocompatibility study, samples were incubated with cells for 24 hours, while for SLN study, cytotoxicity of MDA-MB-231 cells was evaluated after 1 hour incubation at both $37^{\circ} \mathrm{C}$ and $39^{\circ} \mathrm{C}$, respectively. All samples were 


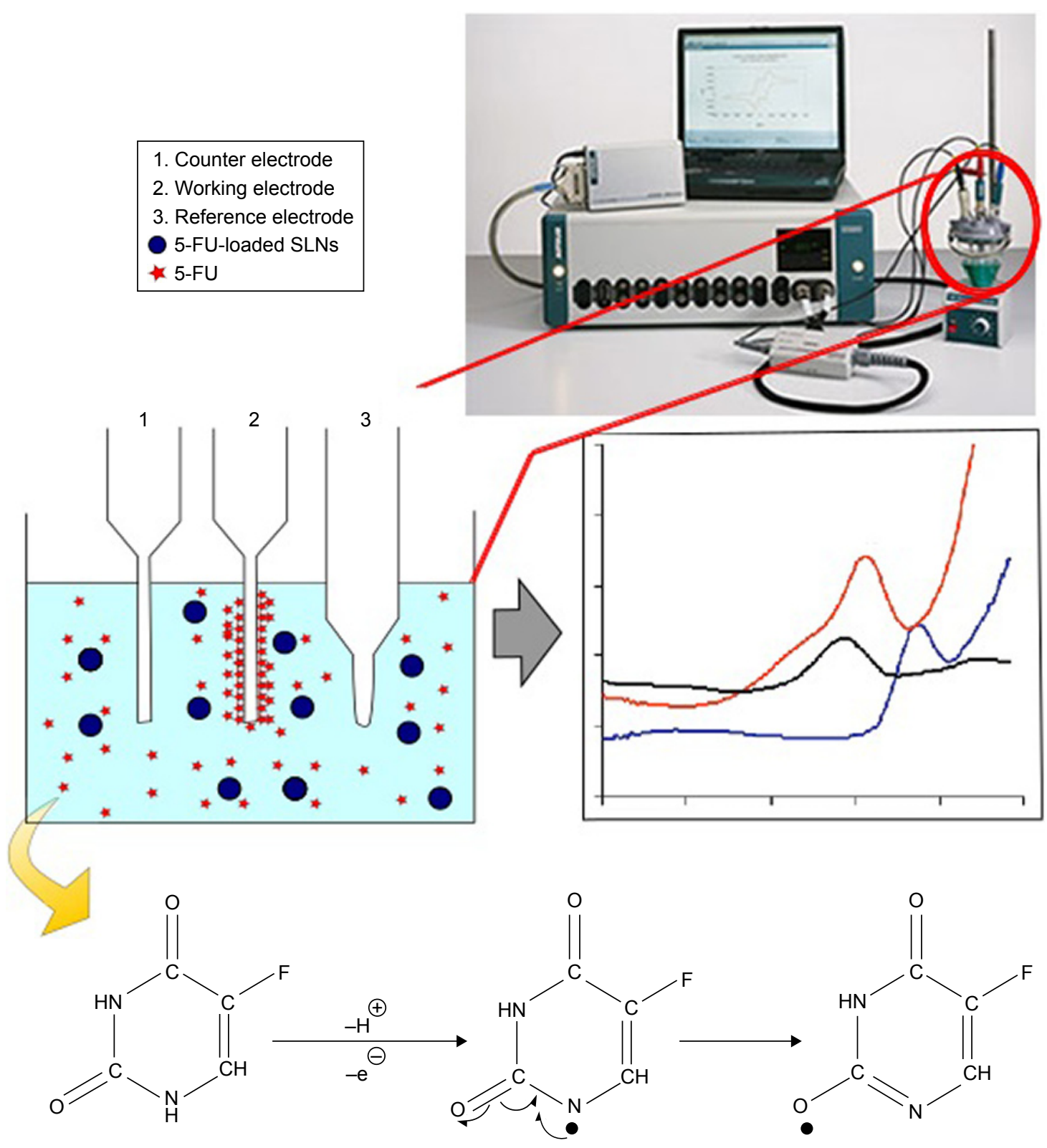

Figure I Schematic presentation of the DPV method for drug release studies.

Notes: 5-FU released from SLNs will undergo oxidation due to applied pulsed voltage. The oxidized 5-FU is accumulated at the working electrode and produces signal proportional to concentration of $5-\mathrm{FU}$ in the dissolution medium.

Abbreviations: DPV, differential pulse voltammetry; 5-FU, 5-fluorouracil; SLN, solid lipid nanoparticle.

sterilized by UV exposure for 20 minutes. All experiments were performed in triplicate, and results are presented as mean \pm SD. Cell viability after exposure to drug-loaded nanoparticles was evaluated by MTS assay.

\section{Statistical analysis}

Experiments were performed in replicates, and results are presented as mean $\pm \mathrm{SD}$. For the comparison of drug release, different models were applied as discussed earlier. For the comparison of cytotoxicity studies, Student's $t$-test and analysis of variance (ANOVA) were used to calculate significance $(p)$, and confidence interval was fixed at $95 \%$.

\section{Results}

\section{Preparation and characterization of TLMs}

TLMs were prepared in varying ratios of solid and liquid fatty acids. Their MPs were determined by DSC studies, and results were plotted against solid lipid proportion (Figure 2A). We observed a linear decrease in MP of all mixtures when the proportion of solid fatty acids in TLMs 
A

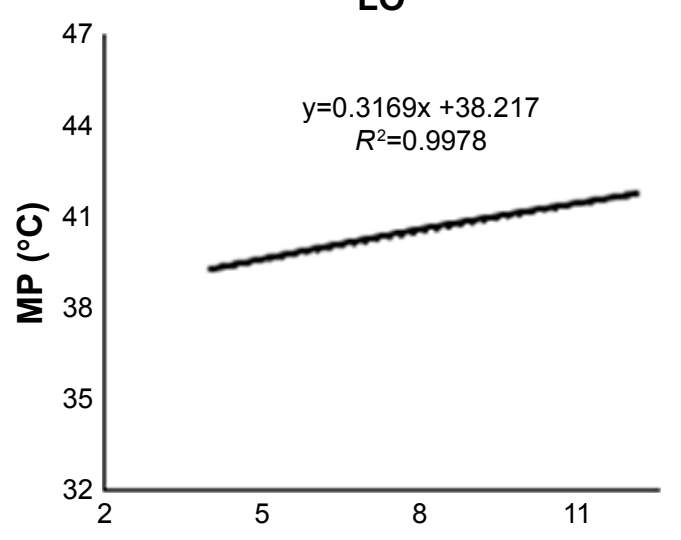

LL

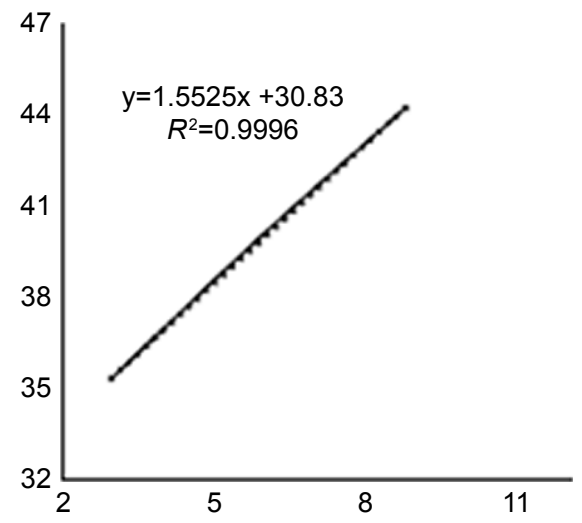

B

\section{Solid lipid proportion}

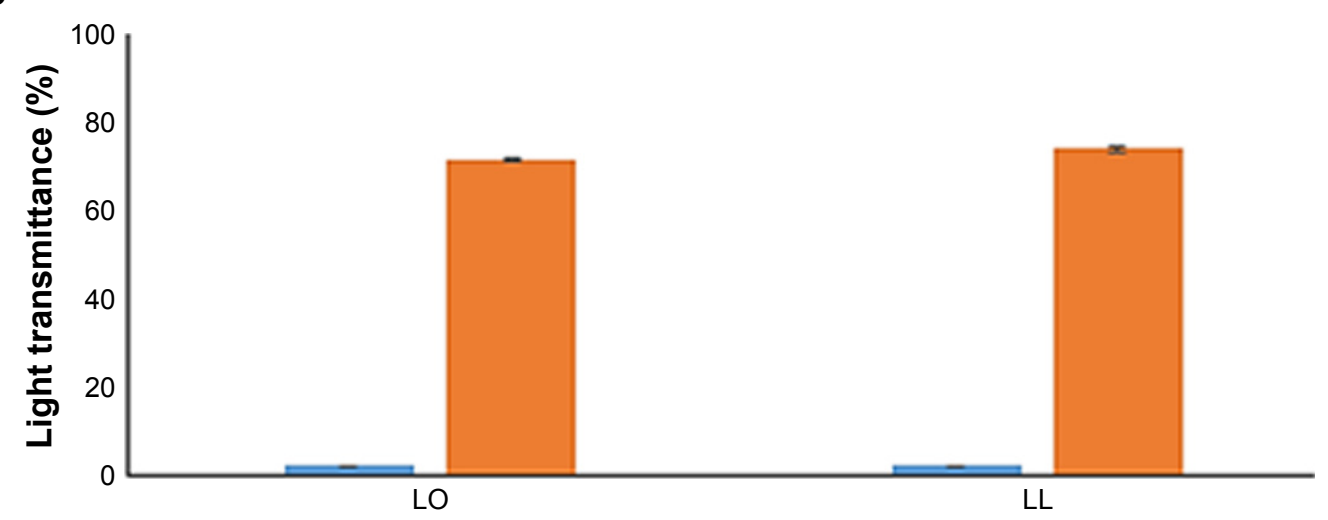

C

\section{Lipid mixtures}

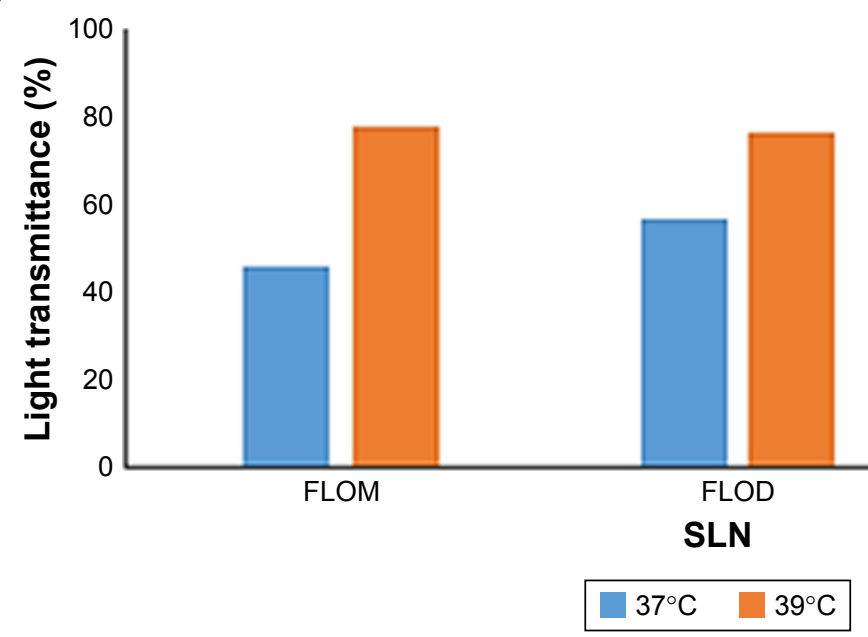

Figure 2 Preparation of TLMs and their characterization.

Note: (A) Standard curve of lipid mixtures LO and LL for selections of lipid mixture with MP of $39^{\circ} \mathrm{C}$, (B) light transmittance from lipid mixtures LO and LL for solid-liquid phase transition, and (C) light transmittance of SLNs prepared from LO (FLOM and FLOD) and LL (FLLD).

Abbreviations: TLM, thermoresponsive lipid mixture; LO, lauric acid and oleic acid; LL, lauric acid and linoleic acid; MP, melting point; SLN, solid lipid nanoparticle; 5-FU, 5-fluorouracil; FLOM, 5-FU-loaded lauric acid and oleic acid nanoparticles prepared with mono-surfactant system; FLOD, 5-FU-loaded lauric acid and oleic acid nanoparticles prepared with double-surfactant system; FLLD, 5-FU-loaded lauric acid and linoleic acid nanoparticles prepared with double-surfactant system; SLN, solid lipid nanoparticle.

decreased with respect to a constant amount of liquid fatty acids. TLMs were identified by straight line equation. The selected TLMs (with MP $\sim 39^{\circ} \mathrm{C}$ ) were found in a molar ratio of 5.26:1 and 2.47:1 for LO and LL, respectively.
These TLMs would melt at $39^{\circ} \mathrm{C}$, which is the desirable temperature for thermoresponsive drug release.

We confirmed thermoresponsive behavior of the TLMs by performing light transmission studies. All TLMs remained 
solid at $37^{\circ} \mathrm{C}$ as negligible light transmission was observed (Figure 2B). However, high light transmission ( $>75 \%$ ) was observed at $39^{\circ} \mathrm{C}$ for both LO and LL, respectively, which indicated conversion of TLM into more transparent liquid phase. ${ }^{17}$

\section{Preparation and characterization of SLNs}

The SLNs were prepared by the HME method, which is a one pot method and uses only water as a solvent in addition to the SLN components. Therefore, the SLNs prepared in this study by just physical mixing of fatty acid can be a safe alternative to other TDDS. The process parameters used in this method were similar to those required for conventional semisolid dosage forms and may be feasible for large-scale production. 5-FU-loaded lauric acid and linoleic acid nanoparticles prepared with mono-surfactant system (FLLM) were unstable and formed a gel-like phase immediately. Therefore, it was not characterized further and removed from the study. Size and zeta potential of SLNs are important due to many reasons. First, they should be small enough to cross the biological barriers such as fenestrated blood vessels with fenestrations or pore size of 50-150 nm. ${ }^{27}$ Second, nanoparticles production should be homogenous for better performance in vitro and in vivo. ${ }^{28}$ On the other hand, zeta potential is important with respect to colloidal stability of the SLNs. Nanoparticles with a zeta potential between 20 and $40 \mathrm{mV}$ will have sufficient repulsion to remain homogeneously dispersed in the aqueous dispersion. ${ }^{29}$ The size of 5-FUloaded lauric acid and oleic acid nanoparticles prepared with mono-surfactant system (FLOM), 5-FU-loaded lauric acid and oleic acid nanoparticles prepared with double-surfactant system (FLOD) and 5-FU loaded lauric acid and linoleic acid nanoparticles prepared with double-surfactant system (FLLD) was $<200 \mathrm{~nm}$ (polydispersity index [PDI] $<0.2$ ), and zeta potential was $<-30 \mathrm{mV}$ in all cases, which is desirable in drug delivery applications (Table 1). We confirmed thermoresponsive nature of as-prepared SLNs by light transmission studies. Light transmission of SLN dispersions was higher at $39^{\circ} \mathrm{C}$ as compared to $37^{\circ} \mathrm{C}$ (Figure $2 \mathrm{C}$ ). This can be attributed to liquid-phase SLNs at $39^{\circ} \mathrm{C}$ that are more transparent than solid SLNs. ${ }^{17}$ No change was observed in the SLN size when stored with plasma or low-concentration PBS. However, they aggregated in $1 \mathrm{M}$ PBS, and the size of FLOM, FLOD and FLLD was increased to 360, 514 and $419 \mathrm{~nm}$, respectively. The higher electrolyte concentration caused reduction in electrostatic repulsion, which resulted in aggregation of SLNs. ${ }^{30}$

\section{Drug loading studies}

The EE of 5-FU with different formulations is given in Table 1. No modification was done to improve EE because it might have affected the thermoresponsive behavior of prepared SLNs. The amount of 5-FU encapsulated in SLNs was sufficient for further analysis.

\section{Transmission electron microscopy}

Transmission electron micrograph reveals that FLOM (Figure 3A), FLOD (Figure 3B), FLLM (Figure 3C) and FLLD (Figure 3D) possess spherical shape and smooth surface. Aggregated nanoparticles of FLLD can also be seen, and, therefore, it was excluded from further studies.

\section{FTIR studies}

The primary amine $(-\mathrm{NH})$ stretching of 5-FU is present between 3,300 and $3,400 \mathrm{~cm}^{-1}$, which indicates that $5-\mathrm{FU}$ was present intact in SLNs. ${ }^{20}$ The low intensity of 5-FU peaks was due to the amount of 5-FU in the SLNs (Figure 4).

\section{Thermoresponsive drug release studies by UV-Vis spectrophotometry}

First, USP type II apparatus was used for dissolution experiment, and the amount of 5-FU released was determined by a UV-Vis spectrophotometer using the calibration curve (Figure 5A). We found that 5-FU release was slow at $37^{\circ} \mathrm{C}$ $(<28 \%)$ and exhibited faster drug release $(>90 \%)$ at $39^{\circ} \mathrm{C}$, which is characteristic of TDDS, such as thermoresponsive liposomes (Figure 5B). ${ }^{8}$ The significantly higher drug release at $39^{\circ} \mathrm{C}$ ( $p$-value $<0.05$ ) confirmed that the SLNs show thermoresponsive drug release at hyperthermic temperature. It can also be seen that the drug release at $39^{\circ} \mathrm{C}$ is similar to the drug release from model OA emulsions used in the study (Figure 5B), thus suggesting that the thermoresponsive drug release from SLNs is due to their existence in the liquid state at hyperthermic temperature. Although TDDS have been reported with targeting temperature of $>41^{\circ} \mathrm{C}$ by using external hyperthermia, we have prepared SLNs to release payload at $39^{\circ} \mathrm{C}$, which is also known as low critical temperature and is more commonly encountered in the clinical setup., ${ }^{2,4}$ Among the three formulations, FLOM showed relatively faster drug release as $100 \%$ drug was released in $<5$ hours. This may be due to the fact that the double surfactant system formed a compact coating that also retarded the release of 5-FU.

\section{Thermoresponsive drug release studies by DPV method}

The DPV method was used for real-time detection of thermoresponsive drug release from SLNs. 5-FU peak was 

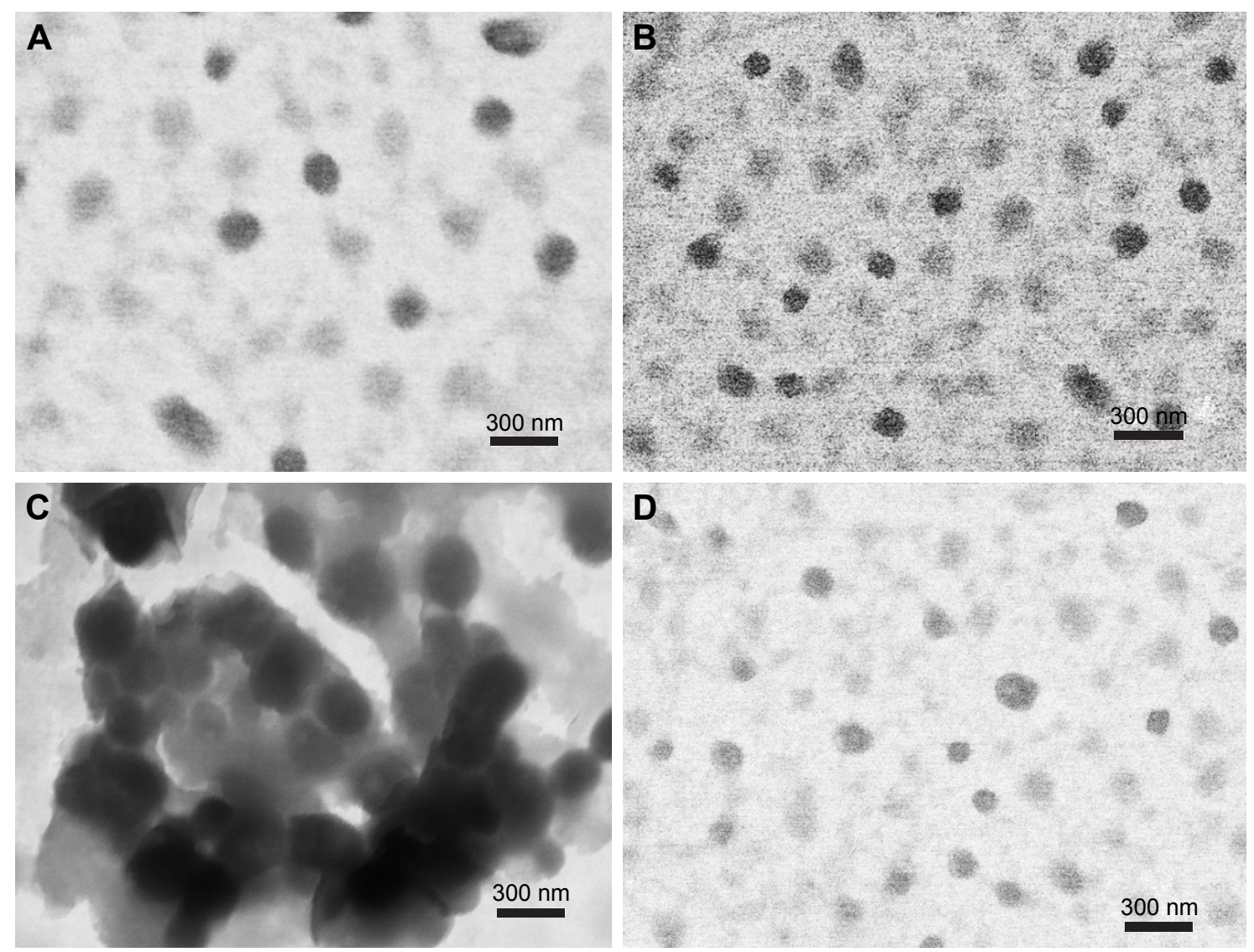

Figure 3 TEM images of (A) FLOM, (B) FLOD, (C) FLLM and (D) FLLD.

Abbreviations: TEM, transmission electron microscopy; FLOM, 5-FU-loaded lauric acid and oleic acid nanoparticles prepared with mono-surfactant system; FLOD, 5-FUloaded lauric acid and oleic acid nanoparticles prepared with double-surfactant system; FLLM, 5-FU-loaded lauric acid and linoleic acid nanoparticles prepared with monosurfactant system; FLLD, 5-FU-loaded lauric acid and linoleic acid nanoparticles prepared with double-surfactant system; 5-FU, 5-fluorouracil.

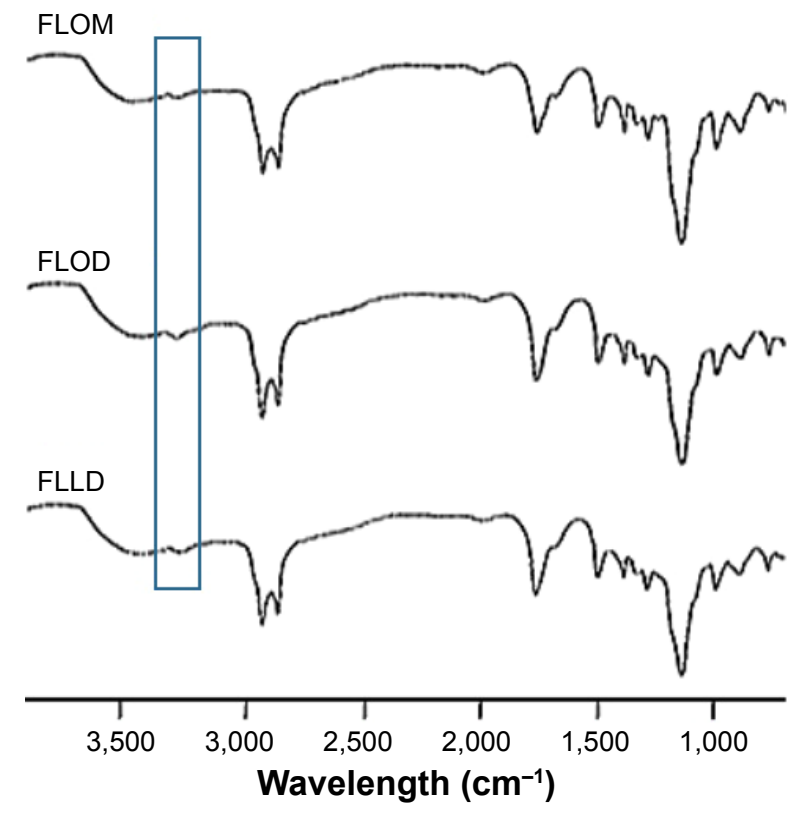

Figure 4 FTIR spectra of FLOM, FLOD and FLLD.

Abbreviations: FTIR, Fourier transform infrared spectroscopy; FLOM, 5-FU-loaded lauric acid and oleic acid nanoparticles prepared with mono-surfactant system; FLOD, 5-FU-loaded lauric acid and oleic acid nanoparticles prepared with doublesurfactant system; FLLD, 5-FU-loaded lauric acid and linoleic acid nanoparticles prepared with double-surfactant system; 5-FU, 5-fluorouracil. observed at $1.15 \mathrm{~V}$, and the difference in peak intensity was recorded at different drug concentrations $(0.05-2.5 \mathrm{mM})$. The DPV method showed linear relation between 5-FU concentration and current measurement by electrodes (Figure 6A), which implies that the DPV method can be used for the detection of this drug using a calibration curve (Figure 6B). In the drug release studies, the DPV method also confirmed thermoresponsive drug release from the SLNs as the sustained drug release pattern at $37^{\circ} \mathrm{C}$ was transformed into faster release at $39^{\circ} \mathrm{C}$ (Figure 6C). DPV is a one pot method and required smaller amount of dissolution media. It also eliminated the need to take samples from the dissolution medium for analysis and subsequent adjustment of the formulas for samples removed. Previously, other novel methods have been suggested to monitor drug release such as Förster resonance energy transfer (FRET). However, FRET is used mainly to measure release of fluorescent drug molecules, ${ }^{31}$ whereas DPV can be used for both fluorescent and nonfluorescent molecules. The drug release profiles obtained by the DPV method corroborates the results of the UV-Vis detection method. Therefore, the DPV method may be used as a 

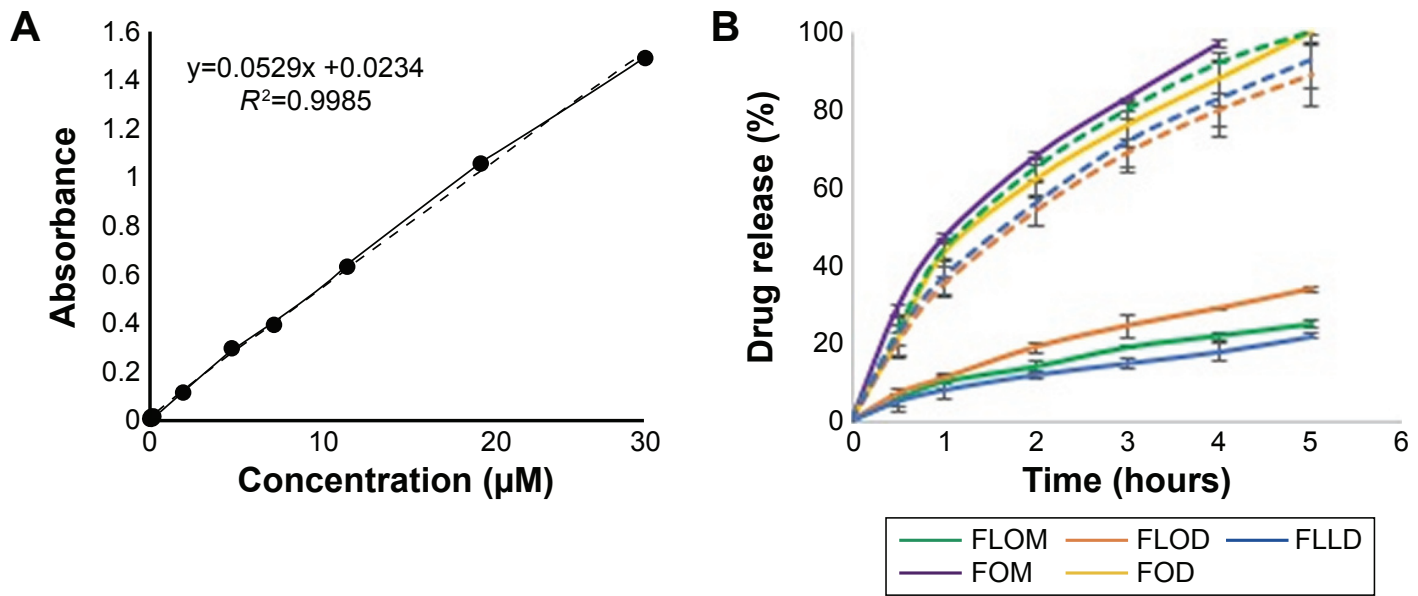

Figure 5 Graphical presentation of the (A) calibration curve of 5-FU prepared by using UV-Vis spectrophotometer and (B) 5 -FU release from SLNs at $37^{\circ} \mathrm{C}$ (solid lines) and $39^{\circ} \mathrm{C}$ (dashed lines) by using USP dissolution apparatus (data $=$ mean $\pm \mathrm{SD}, \mathrm{n}=3$ ).

Abbreviations: 5-FU, 5-fluorouracil; UV-Vis, ultraviolet-visible; SLN, solid lipid nanoparticle; FLOM, 5-FU-loaded lauric acid and oleic acid nanoparticles prepared with mono-surfactant system; FLOD, 5-FU-loaded lauric acid and oleic acid nanoparticles prepared with double-surfactant system; FLLD, 5-FU-loaded lauric acid and linoleic acid nanoparticles prepared with double-surfactant system; FOD, 5-FU-loaded oleic acid microemulsion with double-surfactant system; FOM, 5-FU-loaded oleic acid microemulsion with mono-surfactant system; USP, United States Pharmacopeia.

simple and efficient alternative to conventional dissolution studies for real-time analysis of drug release.

\section{Dissolution kinetic analysis}

In drug release kinetics analysis, all SLN formulations fit better to the zero-order model depicting a constant drug release at $37^{\circ} \mathrm{C}$ (Table 2). It can also be seen that the values of zero-order rate constant are higher at $39^{\circ} \mathrm{C}$, which confirms faster drug release at the hyperthermic temperature. In the Korsmeyer-Peppas model, the value of release exponent " $\mathrm{n}$ " was between 0.5 and 1 at $37^{\circ} \mathrm{C}$, whereas it was $<0.5$ at $39^{\circ} \mathrm{C}$. These results suggested that the 5-FU release from
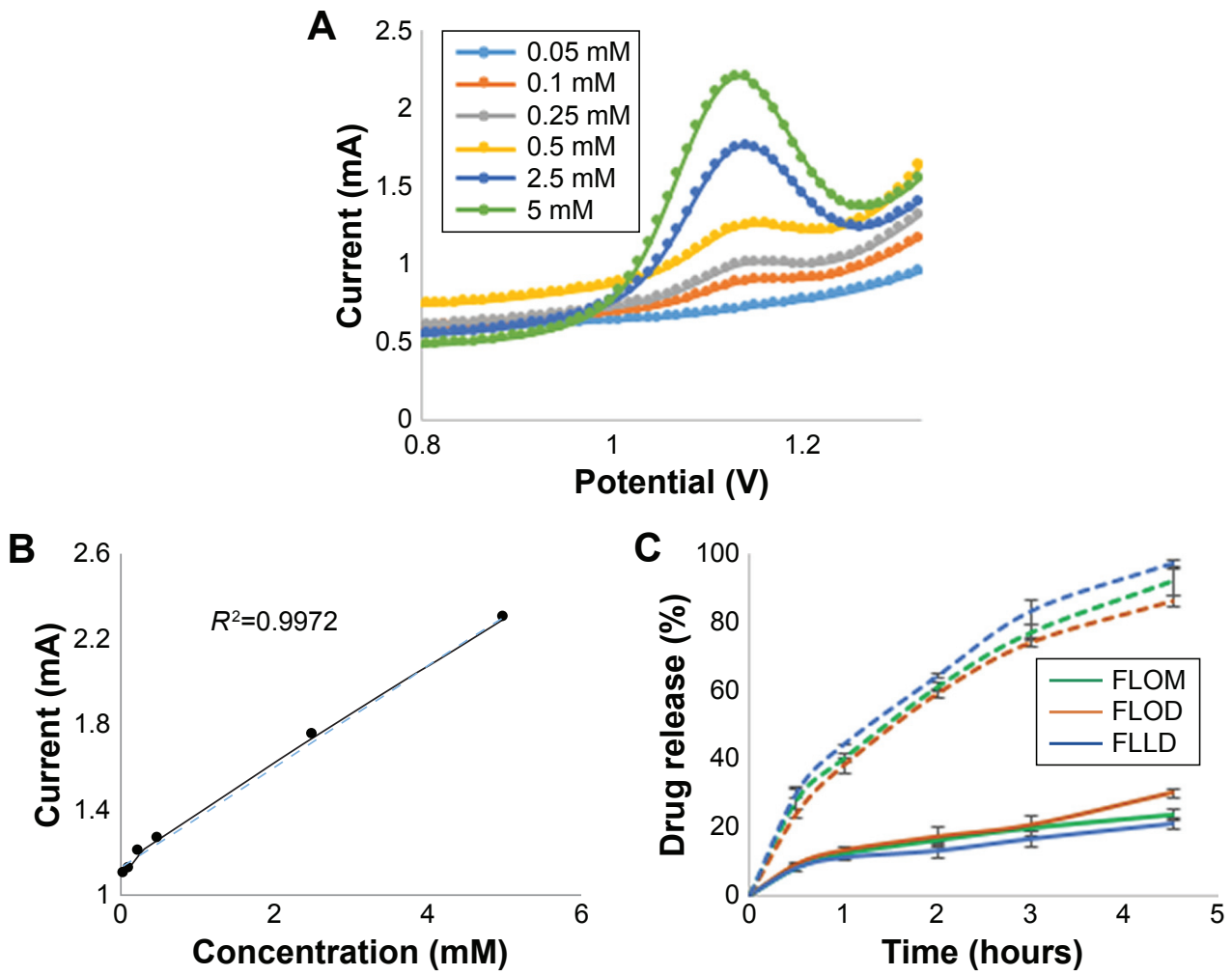

Figure 6 DPV method for the (A) DPV response to different concentrations of 5-FU, (B) calibration curve of 5-FU as plot of drug concentration and measured electric current, and (C) 5 -FU release from SLNs at $37^{\circ} \mathrm{C}$ (solid lines) and $39^{\circ} \mathrm{C}$ (dashed lines) (data = mean $\pm \mathrm{SD}, \mathrm{n}=3$ ).

Abbreviations: DPV, differential pulse voltammetry; 5-FU, 5-fluorouracil; SLN, solid lipid nanoparticle; FLOM, 5-FU-loaded lauric acid and oleic acid nanoparticles prepared with mono-surfactant system; FLOD, 5-FU-loaded lauric acid and oleic acid nanoparticles prepared with double-surfactant system; FLLD, 5-FU-loaded lauric acid and linoleic acid nanoparticles prepared with double-surfactant system. 
Table 2 Kinetic modeling of drug release profiles by data fitting in different models

\begin{tabular}{|c|c|c|c|c|c|c|c|c|c|}
\hline \multirow[t]{2}{*}{ Formulation } & \multicolumn{2}{|c|}{ Zero order } & \multicolumn{2}{|c|}{ First order } & \multicolumn{3}{|c|}{ Korsmeyer-Peppas } & \multicolumn{2}{|c|}{ Higuchi } \\
\hline & $R^{2}$ & $K_{0}$ & $R^{2}$ & $K$ & $R^{2}$ & $K$ & $n$ & $R^{2}$ & $K_{\mathrm{H}}$ \\
\hline \multicolumn{10}{|c|}{ UV-Vis detection method } \\
\hline FLOM37 & 0.9959 & 4.60 & 0.7212 & 0.1574 & 0.9964 & 1.7581 & $0.848 I$ & 0.9927 & 9.4154 \\
\hline FLOD37 & 0.9856 & 4.6667 & 0.8257 & 0.1013 & 0.9931 & 2.26 & 0.5366 & 0.9975 & 14.2620 \\
\hline FLLD37 & 0.9918 & 5.75 & 0.9604 & 0.312 & 0.9999 & 1.171 & 0.8177 & 0.9933 & 8.0541 \\
\hline FLOM39 & 0.9695 & 17.8 & 0.8524 & 0.1989 & 0.9996 & 2.1983 & 0.471 & 0.9973 & 40.2726 \\
\hline FLOD39 & 0.9665 & 17 & 0.8453 & 0.2039 & 0.9995 & 2.1427 & 0.488 & 0.9975 & 38.7371 \\
\hline FLLD39 & 0.9695 & 19.6 & 0.823 & 0.2053 & 0.9948 & 2.1932 & 0.4297 & 0.99 & 45.3742 \\
\hline FOM37 & 0.9728 & 21.5 & 0.8937 & 0.2416 & 0.9949 & 1.9567 & 0.5 & 0.9982 & 47.8758 \\
\hline FOD37 & 0.9896 & 20.2 & 0.8229 & 0.211 & 0.9998 & 2.056 & 0.5 & 0.9922 & 43.8881 \\
\hline \multicolumn{10}{|c|}{ Electrochemical detection method } \\
\hline FLOM37 & 0.9887 & 4.52 & 0.8873 & 0.2021 & 0.9959 & 1.1539 & 0.4836 & 0.9972 & 11.3805 \\
\hline FLOD37 & 0.9858 & 4.5731 & 0.9558 & 0.2351 & 0.9769 & 1.1817 & 0.5765 & 0.9601 & 12.7262 \\
\hline FLLD37 & 0.9922 & 3.615 & 0.9497 & 0.3004 & 0.9785 & 1.2742 & 0.528 & 0.9796 & 10.1807 \\
\hline FLOM39 & 0.9817 & 25.4 & 0.9008 & 0.3718 & 0.998 & 1.1828 & 0.5342 & 0.9975 & 42.1113 \\
\hline FLOD39 & 0.9687 & 24 & 0.8606 & 0.4215 & 0.9928 & 1.2264 & 0.5568 & 0.992 & 39.385 \\
\hline FLLD39 & 0.98 & 23.3810 & 0.9093 & 0.376 & 0.9975 & 1.1539 & 0.5288 & 0.9954 & 45.0523 \\
\hline
\end{tabular}

Notes: $R^{2}$, coefficient of determination; $K_{0}$, zero order rate constant; $K$, rate constant in first order and Korsmeyer-Peppas model; $K_{H}$, Higuchi rate constant; and $n$, release exponent of Korsmeyer-Peppas model. In the "Formulation" column, 37 and 39 represent temperature $\left({ }^{\circ} \mathrm{C}\right)$ of drug release studies.

Abbreviations: UV-Vis, ultraviolet-visible; FLOM, 5-FU-loaded lauric acid and oleic acid nanoparticles prepared with mono-surfactant system; FLOD, 5-FU-loaded lauric acid and oleic acid nanoparticles prepared with double-surfactant system; FLLD, 5-FU-loaded lauric acid and linoleic acid nanoparticles prepared with double-surfactant system; 5-FU, 5-fluorouracil; FOD, 5-FU-loaded oleic acid microemulsion with double-surfactant system; FOM, 5-FU-loaded oleic acid microemulsion with mono-surfactant system.

the SLNs was anomalous at $37^{\circ} \mathrm{C}$ and diffusion controlled at $39^{\circ} \mathrm{C}$. In SLNs, the aqueous dissolution medium has slow penetration in lipids, which leads to sustained release of drug over prolonged period of time. ${ }^{14}$ Furthermore, the surfactant coating the SLN surface may be removed and diffuse to the surrounding aqueous phase. The pores caused by the removal of the surfactant molecules may facilitate the penetration of water corresponding to the erosion-controlled release mechanism, although it is a slow process. ${ }^{14}$ In this situation, drug release mechanism will be anomalous, ie, superimposition of diffusion and erosion drug release. At $39^{\circ} \mathrm{C}$, the drug release was faster and the diffusion would prevail as drug release mechanism. In the DPV method, the value of " $n$ " was between 0.5 and 1 at both temperatures, suggesting that anomalous drug release occurs at both temperatures, although "n" values were closer to the diffusion region at $39^{\circ} \mathrm{C}$. Similarly, all formulations showed good fit to the Higuchi model at both temperatures, thus suggesting that the diffusion was the predominant mechanism for the release of the 5 -FU.

Comparison of both dissolution methods is presented in terms of difference and similarity factors (Table 3 ). In all cases, the values of difference factors were $<15$ and values of similarity factors were $>50$. This depicts that drug release profiles obtained by the UV-Vis spectrophotometer and DPV are similar. The agreement in the results of both methods proves that the DPV method can be used for real-time monitoring of drug release from nanoparticles.

\section{Cytotoxicity studies}

The cytotoxicity studies were performed against HGF cells for 24 hours at $37^{\circ} \mathrm{C}$, and the results are shown in Figure $7 \mathrm{~A}$. The results indicated that blank SLNs showed good compatibility ( $>90 \%$ cell viability) even at a high dose, whereas 5-FU-loaded SLN formulations showed dose-dependent cytotoxicity. Among all the 5-FU-loaded nanoparticles, FLOM generated relatively higher cytotoxicity, but cell viability was still $>80 \%$ after 24 hours. Nevertheless, the difference in cytotoxicity of FLOM, FLOD and FLLD was insignificant $(p>0.05)$. On the other hand, all formulations showed thermoresponsive cytotoxicity against the MDA-MB-231 cells (Figure 7B). Although low cytotoxicity was observed at $37^{\circ} \mathrm{C}(2 \%-8 \%)$, it was higher at

Table 3 Similarity factors and difference factors of drug release profiles by UV-Vis spectrophotometer and the DPV method

\begin{tabular}{lll}
\hline Formulation & $\begin{array}{l}\text { Difference } \\
\text { factor }\left(f_{1}\right)\end{array}$ & $\begin{array}{l}\text { Similarity } \\
\text { factor }\left(f_{2}\right)\end{array}$ \\
\hline FLOM37 & 8 & 90 \\
FLOD37 & 12 & 83 \\
FLLD37 & 5 & 95 \\
FLOM39 & 5 & 78 \\
FLOD39 & 5 & 77 \\
FLLD39 & 6 & 73 \\
\hline
\end{tabular}

Notes: In the "Formulation" column, 37 and 39 represent temperature $\left({ }^{\circ} \mathrm{C}\right)$ of drug release studies.

Abbreviations: UV-Vis, ultraviolet-visible; DPV, differential pulse voltammetry; FLOM, 5-FU-loaded lauric acid and oleic acid nanoparticles prepared with monosurfactant system; FLOD, 5-FU-loaded lauric acid and oleic acid nanoparticles prepared with double-surfactant system; FLLD, 5-FU-loaded lauric acid and linoleic acid nanoparticles prepared with double-surfactant system; 5-FU, 5-fluorouracil. 

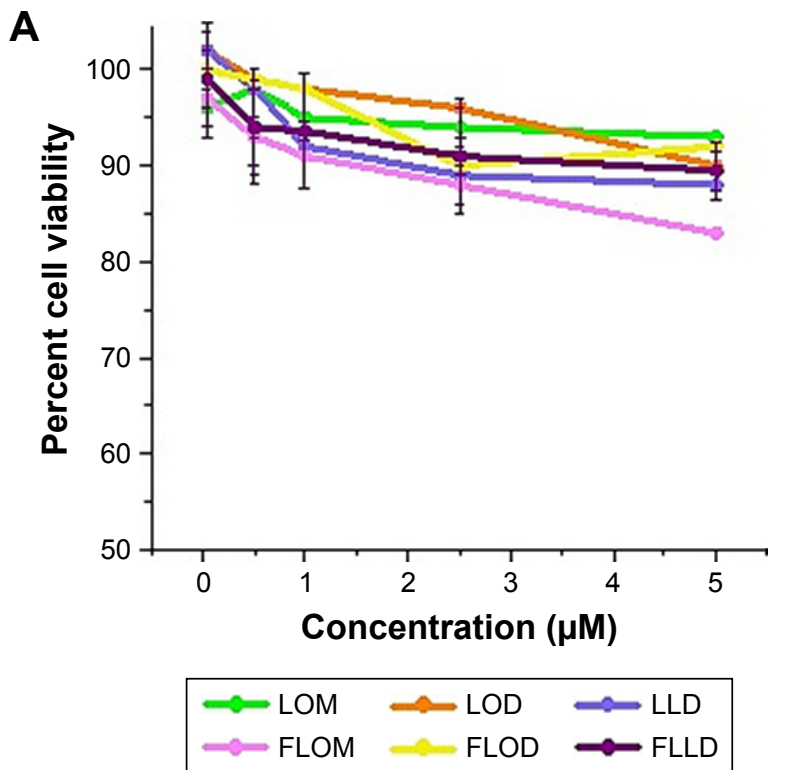
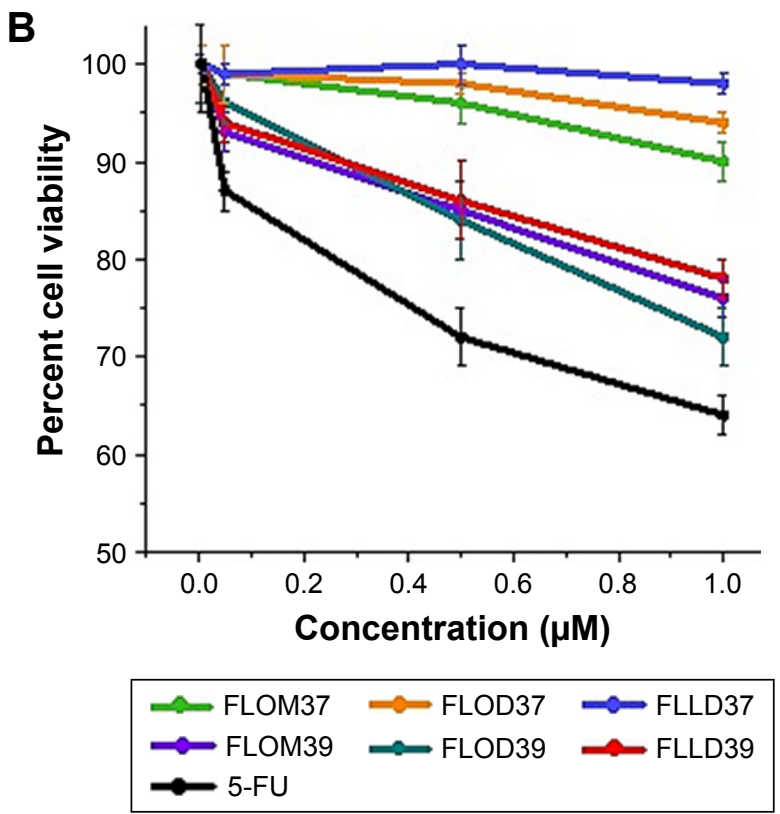

Figure 7 Biocompatibility and cytotoxicity of the (A) blank and 5-FU-loaded SLNs against HGF cells after 24 hours and (B) 5-FU-loaded SLNs against breast cancer cells (MDA-MB-23I) after I hour at $37^{\circ} \mathrm{C}$ and $39^{\circ} \mathrm{C}$ (data $=$ mean $\pm S D, n=3$ ).

Note: (B) 37 and 39 represent temperature $\left({ }^{\circ} \mathrm{C}\right)$ of drug release studies.

Abbreviations: 5-FU, 5-fluorouracil; SLN, solid lipid nanoparticle; HGF, human gingival fibroblast; FLOM, 5-FU-loaded lauric acid and oleic acid nanoparticles prepared with mono-surfactant system; FLOD, 5-FU-loaded lauric acid and oleic acid nanoparticles prepared with double-surfactant system; FLLD, 5-FU-loaded lauric acid and linoleic acid nanoparticles prepared with double-surfactant system; LOM, lauric acid and oleic acid nanoparticles prepared with mono-surfactant system; LOD, lauric acid and oleic acid nanoparticles prepared with double-surfactant system; LLD, lauric acid and linoleic acid nanoparticles prepared with double-surfactant system.

$39^{\circ} \mathrm{C}(22 \%-28 \%)$ after 1 hour exposure. The significantly higher cytotoxicity $(p<0.05)$ at hyperthermic temperature was due to the higher amount of 5-FU released at certain temperature. 5-FU belongs to antimetabolites family of anticancer drugs. It exerts cytotoxic effects by blocking nucleic acid synthesis, ie, by incorporation of its metabolites in RNA and DNA and by inhibiting thymidylate synthase. ${ }^{32}$ In addition, the cytotoxicity of SLNs was lower than the cytotoxicity induced by the same concentration of 5 -FU solution (Figure 7B). The reason for this observation was that the 5-FU was released from SLNs over time and the whole drug was not available to induce cytotoxicity during 1-hour study period. The cytotoxicity studies were conducted for 1 hour at hyperthermic temperature in accordance with the generally used protocol for thermotherapy of cancer. ${ }^{7,33}$

\section{Conclusion}

This article reports a quick and reliable method for the preparation of SLNs by physical mixing of solid and liquid fatty acids. The thermoresponsive behavior of lipids and as-prepared SLNs was confirmed by higher light transmission from the liquid phase at $39^{\circ} \mathrm{C}$. Drug release studies showed that 5 -FU release was higher at $39^{\circ} \mathrm{C}$ due to the presence of liquid phase than that of $37^{\circ} \mathrm{C}$. Further proof of thermoresponsive drug release from SLNs was obtained by devising a DPV method for real-time electrochemical detection of 5-FU, which also confirmed thermoresponsive drug release at $39^{\circ} \mathrm{C}$. A model-independent approach confirmed the agreement in results of spectrophotometry and DPV methods. In model-dependent analysis, drug release was found to be diffusion controlled at $39^{\circ} \mathrm{C}$ as predicted by good fit to Higuchi and Korsmeyer-Peppas model $(\mathrm{n}<0.5)$. SLNs were biocompatible to HGF cells and exhibited 5-FU dose-dependent cytotoxicity to MDA-MB-231 breast cancer cells. Two to three times higher cytotoxicity was achieved against cancer cells at $39^{\circ} \mathrm{C}$, which also suggested thermoresponsive targeting by using SLNs. Hence, this study provided a thermoresponsive targeting platform by using SLNs that may be simple, economical and a safe alternative to other thermoresponsive DDS.

\section{Acknowledgments}

The authors acknowledge the contribution of Naveed Akhter and Anum Munawar during different experiments. The authors would also like to thank the National Institute of Biotechnology and Genetic Engineering, Pakistan; The Islamia University of Bahawalpur, Pakistan, and Northeastern University, MA, USA, for providing research facilities. MR also availed two scholarships from the Higher Education Commission of Pakistan under the Indigenous PhD Scholarship (Pin: 213-53204-2BM2-034) and International 
Research Support Initiative Program (Pin: IRSIP 32 PSc 15) during this study.

\section{Disclosure}

The authors report no conflicts of interest in this work.

\section{References}

1. Dou Y, Hynynen K, Allen C. To heat or not to heat: challenges with clinical translation of thermosensitive liposomes. J Control Release. 2017; 249:63-73.

2. Madni A, Sarfraz M, Rehman M, et al. Liposomal drug delivery: a versatile platform for challenging clinical applications. J Pharm Pharm Sci. 2014;17(3):401-426.

3. Lu T, ten Hagen TL. Inhomogeneous crystal grain formation in DPPCDSPC based thermosensitive liposomes determines content release kinetics. J Control Release. 2016;247:64-72.

4. Al-Ahmady Z, Kostarelos K. Chemical components for the design of temperature-responsive vesicles as cancer therapeutics. Chem Rev. 2016; 116(6):3883-3918.

5. Bulbake U, Doppalapudi S, Kommineni N, Khan W. Liposomal formulations in clinical use: an updated review. Pharmaceutics. 2017;9(2):e12.

6. Brezaniova I, Hruby M, Kralova J, et al. Temoporfin-loaded 1-tetradecanol-based thermoresponsive solid lipid nanoparticles for photodynamic therapy. J Control Release. 2016;241:34-44.

7. Christensen KY, Vizcaya D, Richardson H, Lavoué J, Aronson K, Siemiatycki J. Risk of selected cancers due to occupational exposure to chlorinated solvents in a case-control study in Montreal. J Occup Environ Med. 2013;55(2):198-208.

8. Lokerse WJ, Kneepkens EC, ten Hagen TL, Eggermont AM, Grüll H, Koning GA. In depth study on thermosensitive liposomes: optimizing formulations for tumor specific therapy and in vitro to in vivo relations. Biomaterials. 2016;82:138-150.

9. Greish K. Enhanced permeability and retention (EPR) effect for anticancer nanomedicine drug targeting. Cancer Nanotechnol. 2010;624:25-37.

10. Priano L, Esposti D, Esposti R, et al. Solid lipid nanoparticles incorporating melatonin as new model for sustained oral and transdermal delivery systems. J Nanosci Nanotechnol. 2007;7(10):3596-3601.

11. Doktorovova S, Souto EB, Silva AM. Nanotoxicology applied to solid lipid nanoparticles and nanostructured lipid carriers-a systematic review of in vitro data. Eur J Pharm Biopharm. 2014;87(1):1-18.

12. Hao J, Wang X, Bi Y, et al. Fabrication of a composite system combining solid lipid nanoparticles and thermosensitive hydrogel for challenging ophthalmic drug delivery. Colloids Surf B Biointerfaces. 2014;114:111-120.

13. Rehman M, Asadullah Madni WSK, Ihsan A, Khan MI, Chaudhary OJ. Sustained release solid lipid nanoparticles of simultaneously loaded hydrophobic drug, diacerein and gold nanoparticles and their potential for thermoresponsive flux. Paper presented at: Macro 2014; 2014; Chiang Mai, Thailand.

14. Rehman M, Asadullah Madni AI, Khan WS, et al. Solid and liquid lipid-based binary solid lipid nanoparticles of diacerein: in vitro evaluation of sustained release, simultaneous loading of gold nanoparticles, and potential thermoresponsive behavior. Int J Nanomedicine. 2015; 10:2805.
15. ud Din F, Mustapha O, Kim DW, et al. Novel dual-reverse thermosensitive solid lipid nanoparticle-loaded hydrogel for rectal administration of flurbiprofen with improved bioavailability and reduced initial burst effect. Eur J Pharm Biopharm. 2015;94:64-72.

16. Shah A, Nosheen E, Zafar F, et al. Photochemistry and electrochemistry of anticancer uracils. J Photochem Photobiol B. 2012;117:269-277.

17. Helgason T, Salminen H, Kristbergsson K, McClements DJ, Weiss J. Formation of transparent solid lipid nanoparticles by microfluidization: influence of lipid physical state on appearance. J Colloid Interface Sci. 2015;448:114-122.

18. Müller RH, Mäder K, Gohla S. Solid lipid nanoparticles (SLN) for controlled drug delivery-a review of the state of the art. Eur J Pharm Biopharm. 2000;50(1):161-177.

19. Harsha S. Pharmaceutical suspension containing both immediate/ sustained-release amoxicillin-loaded gelatin nanoparticles: preparation and in vitro characterization. Drug Des Devel Ther. 2013;7:1027-1033.

20. Coates J. Interpretation of infrared spectra, a practical approach. Encycl Anal Chem. Epub 2000 Sep 15.

21. Khan MI, Madni A, Peltonen L. Development and in-vitro characterization of sorbitan monolaurate and poloxamer 184 based niosomes for oral delivery of diacerein. Eur J Pharm Sci. 2016;95:88-95.

22. Farooq M, Ahmad M, Madni A, Hanif M, Khan MR. Development and evaluations of aceclofenac microcapsules for colon-targeted delivery: an in vitro study. Adv Polym Technol. 2015;34(2):e21482.

23. Luo Y, Kirker KR, Prestwich GD. Cross-linked hyaluronic acid hydrogel films: new biomaterials for drug delivery. J Control Release. 2000;69(1):169-184.

24. Wang Y, Li X, Wang L, Xu Y, Cheng X, Wei P. Formulation and pharmacokinetic evaluation of a paclitaxel nanosuspension for intravenous delivery. Int J Nanomedicine. 2011;6:1497-1507.

25. US Department of Health and Human Services; Food and Drug Administration; Center for Drug Evaluation and Research (CDER). Guidance for Industry: Dissolution Testing of Immediate Release Solid Oral Dosage Forms [1997]. Available from: https:/www.fda.gov/downloads/ drugs/guidances/ucm070237.pdf. Accessed October 25, 2017.

26. Barzegar-Jalali M, Adibkia K, Valizadeh H, et al. Kinetic analysis of drug release from nanoparticles. J Pharm Pharm Sci. 2008;11(1):167-177.

27. Sutariya VB, Pathak Y. Biointeractions of Nanomaterials. Boca Raton, FL: CRC Press; 2014.

28. Umair M, Javed I, Rehman M, et al. Nanotoxicity of inert materials: the case of gold, silver and iron. J Pharm Pharm Sci. 2016;19(2):161-180.

29. Zhao L, Zhu B, Jia Y, Hou W, Su C. Preparation of biocompatible carboxymethyl chitosan nanoparticles for delivery of antibiotic drug. Biomed Res Int. 2013;2013:e236469.

30. Freitas C. Stability determination of solid lipid nanoparticles (SLN TM) in aqueous dispersion after addition of electrolyte. J Microencapsul. 1999;16(1):59-71.

31. Tang J, Kong B, Wu H, et al. Carbon nanodots featuring efficient FRET for real-time monitoring of drug delivery and two-photon imaging. Adv Mater Deerfield. 2013;25(45):6569-6574.

32. Longley DB, Harkin PD, Johnston PG. 5-fluorouracil: mechanism of action and clinical strategies. Nat Rev Cancer. 2003;3:330-338.

33. Swenson CE, Haemmerich D, Maul DH, Knox B, Ehrhart N, Reed RA. Increased duration of heating boosts local drug deposition during radiofrequency ablation in combination with thermally sensitive liposomes (ThermoDox) in a porcine model. PLoS One. 2015;10(10):e0139752.

International Journal of Nanomedicine

\section{Publish your work in this journal}

The International Journal of Nanomedicine is an international, peerreviewed journal focusing on the application of nanotechnology in diagnostics, therapeutics, and drug delivery systems throughout the biomedical field. This journal is indexed on PubMed Central, MedLine, CAS, SciSearch $\AA$, Current Contents ${ }^{\circledR} /$ Clinical Medicine,

\section{Dovepress}

Journal Citation Reports/Science Edition, EMBase, Scopus and the Elsevier Bibliographic databases. The manuscript management system is completely online and includes a very quick and fair peer-review system, which is all easy to use. Visit http://www.dovepress.com/ testimonials.php to read real quotes from published authors. 